$\underline{\underline{\beta}}=$

\title{
Biopsy proven fibroadenomas- how frequent is pathologies other than fibroadenomas post-excision?
}

\author{
Tajudeen A. Wahab *, Hanna Uwakwe, Rilwan Aransi, Humuyan Khan, Maher Jumah \\ Elm Breast Care Centre, Barking, Havering and Redbridge University Hospitals NHS Trust, King George Hospital. London \\ *Corresponding author E-mail: oroki40@yahoo.com
}

\begin{abstract}
Introduction: Breast fibroadenoma (FA) is a common benign tumour diagnosed usually through 'Triple Assessment'. FA that is large, symptomatic or exhibit interval growth is recommended for excision. Some patients question the need for excision if preoperative assessment concluded FA while others want to be absolutely sure that the 'FA' that is not excised and managed conservatively is absolutely a FA. The aim of the present study is to ascertain the magnitude of unexpected post-excision pathology when a biopsy proven FA is excised according to the current UK guideline.

Materials and Method: Retrospectively analysis of patients who underwent surgery for FA excision over a 3 year period. Data on patient demographics, ultrasound findings and size at diagnosis, any interval change in size, indication for excision were collected and pathology pre- and post-excision compared.

Result: Of the 276 FA excised in 258 patients in 3years, a preoperative diagnosis of FA was confirmed in 251 cases while 25 breast lumps were excised based on the benign features on examination and imaging. The post excision histopathology confirmed the preoperative diagnosis of FA in $264(95.7 \%)$, phyllodes in $8(3 \%)$ and $4(1.3 \%)$ other pathologies.

Conclusion: $4.3 \%$ of FA excised in our specialist unit after Triple Assessment returns pathology other than FA post-excision, mostly benign phyllodes. FAs should be managed conservatively after triple assessment but surgery should be offered if there is still any doubt as there is no absolute guarantee that the presumed FA is actually a FA. For medicolegal reasons and culture of openness, patients should be aware of this possibility especially if FA is managed conservatively.
\end{abstract}

Keywords: Benign Breast lump; Benign Phyllodes Tumor; Breast lump excision; Fibroadenoma; Triple Assessment.

\section{Introduction}

Breast fibroadenoma (FA) is a common benign tumour in premenopausal women that frequently presents as a slowly growing, well defined, mobile lump (1). Currently, diagnosis is through a 'Triple Assessment' which consists of clinical examination, imaging (ultrasound or mammogram) and pathology (fine needle aspiration or core biopsy). Needle biopsies are performed under image guidance to ensure the lesion is accurately targeted. FA may either remain static, continue to grow or decrease in size. Some do disappear especially after menopause (2). In the UK and according to the national guidelines, it not necessary or mandatory to biopsy a FA in patients younger than 25 years of age if the ultrasound scan show typical features of a benign lesion according to the Stavros Criteria [3] [4]. If a diagnosis of a FA is established, it can be managed conservatively without a need for excision and patients can be reassured and discharged (5). Indications for removal of FA are largely because of size $(>3 \mathrm{~cm})$, significant interval increase in size (growing FA), at the request of the patients due to symptoms, or discordance on clinical findings and histology report (5).

In our practice, some patients request for excision despite preoperative confirmation of FA mostly because of the fear breast cancer or 'other' pathologies, while others question the recommendation for excision based on the FA size after a preoperative diagnosis of its benign nature had been confirmed.
The aim of the present study is to ascertain the magnitude of unexpected post-excision pathology when a biopsy proven FA is excised according to the current UK guideline.

\section{Materials and method}

All patients who underwent excision of fibroadenomas in our breast unit over a three-year period were included and analysed retrospectively. Data were extracted from PACs (radiology), histopathology reports, Multidisciplinary Team (MDT) meetings outcome and patients' electronic record. Data collected include the patient demographics, ultrasound findings and FA size at diagnosis, any interval change in size, indication for excision, pathology pre- and post-excision. Descriptive analysis of the above variables was done while the statistical analysis was performed out using Excel 2010 (Microsoft Corporation, Redmond, WA, USA).

\section{Result}

There were 276 FA excised in 258 patients over a $3 y r s$ period of study, giving an average of 92 FA excised each year. The patients' ages range from 15 to 79 years with $41.75 \%$ of the patients in age range 21-30years (Figure 1) 


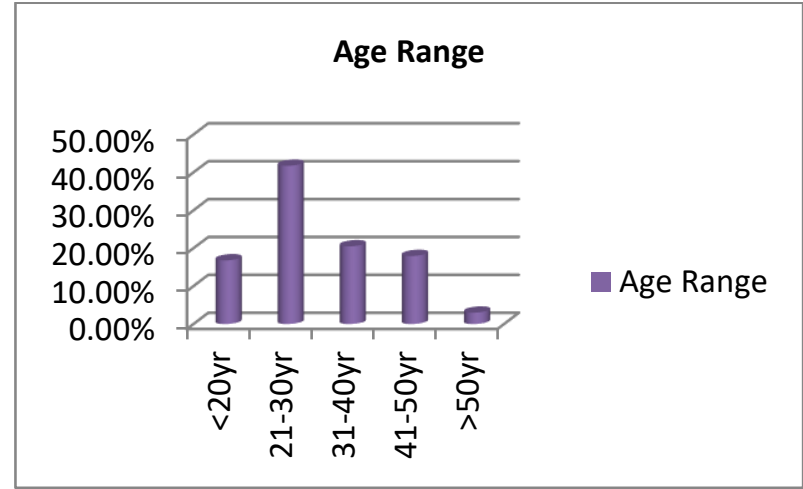

Fig. 1: Patients' Age Groups.

A diagnosis of fibroadenoma was confirmed on histopathology after triple assessment preoperative in 251 FA. Twenty-three breast lumps were excised based on the benign features on clinical examination and imaging only. These were mostly young patients that are less than 25 years of age. A patient had excision of bilateral FA after refusing a preoperative biopsy claiming she has had the lumps for many years and was fairly certain of their noncancerous nature.

The post excision histopathology confirmed the preoperative diagnosis of FA in $264(95.7 \%)$, phyllodes in $8(3 \%)$ and $4(1.3 \%)$ other pathologies (Table.1).

Table 1: Post Excision Pathologies. PASH- Pseudoangiomatous Stromal Hyperplasia

\begin{tabular}{llll}
\hline Pathology & No & $\%$ & Total \\
\hline FA & 264 & 95.7 & 264 \\
FA + DCIS & 1 & 0.3 & 265 \\
Benign Phyllodes & 8 & 3 & 273 \\
PASH & 1 & 0.3 & 274 \\
Fibrocystic changes (FCC) & 1 & 0.3 & 275 \\
Harmatoma & 1 & 0.3 & 276 \\
\hline
\end{tabular}

In about $60 \%$ of our case, the post excision pathology size is bigger than their preoperative USS size by at least $3 \mathrm{~mm}$ (Figure 2).

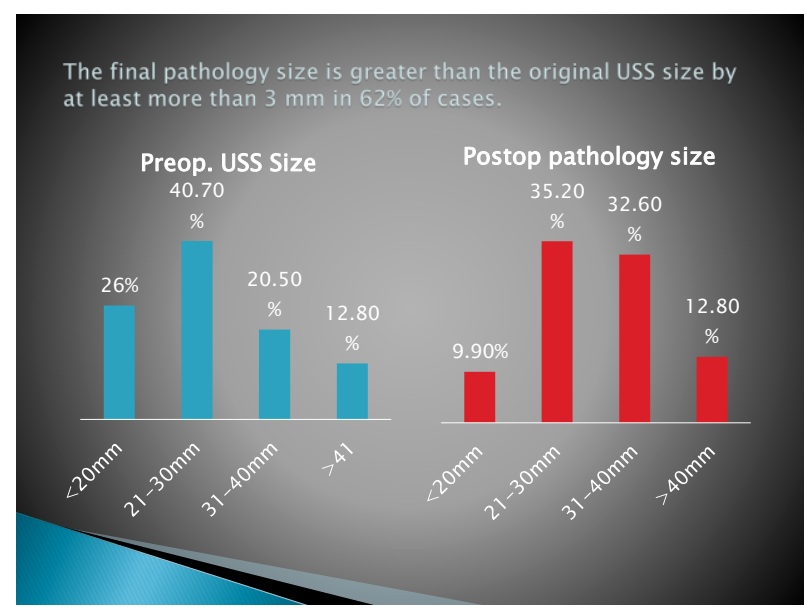

Fig. 2: Preoperative USS and Post Excision Pathological Size.

\section{Discussion}

Histologically, FA is composed of both epithelial and stromal components and is commonly classified as either simple FA or complex FA. A FA containing cysts greater than $3 \mathrm{~mm}$, sclerosing adenosis, epithelial calcifications, or papillary apocrine metaplasia is regarded as complex (6). Women with complex fibroadenomas are believed to have a significantly greater risk of breast cancer than those with simple ones, especially in the presence of family history of breast cancer [6]. A more recent study however suggested that breast cancer risk in patients with FA are similar when compared to benign breast disease and that the presence of com- plex FA does not increased risk of breast cancer on its own[7]. The apparent risk of breast cancer attributed to FA has more to do with the degree of epithelial proliferation present within or adjacent to the FA, rather than with the mere presence of FA. This could be a proliferative disease without atypia [PDWA], or atypical hyperplasia (7). When FA are of large size with increased stroma cellularity and contains epithelial cleft, it needs to be carefully distinguished from benign phyllodes tumours which tend to grow faster, bigger and occurs predominantly in middle age range 40-50 years (8). The peak incidence for FA occurs about a decade earlier than that of benign phyllodes [8]. Eight (3\%) of our excised FA were benign phyllodes following excision. Comparing the characteristics of patients whose histology was PT post excision with those with FA confirmed post excision showed considerable overlap in the age, tumour size and the finds on USS and mammogram. Lee et al (9) found 9\% of their biopsy-proven FA excised because of size $(>2 \mathrm{~cm})$ or interval growth to be phyllodes tumours following excision. Even though there is no clinical evidence that FA turns into breast cancer, there are reports of foci of different histologic types of breast cancer in some excised with the rate varying from $0.002 \%$ to $0.125 \%$ in fibroadenoma specimens [10]. There is only one $(0.3 \%)$ case FA with DCIS in our study which supports a very low incidence of unexpected cancer in excised FA if a preoperative triple assessment has been performed. A retrospective review of 2062 image guided core biopsy proven FA found 12 cases [ $0.58 \%$ ] that demonstrated atypia, in situ or invasive cancer either within or adjacent to the FA[11].

FA size is one of the indications for advising excision even when the diagnosis has been confirmed on biopsy. It is general believed that a FA grows to between $2-3 \mathrm{~cm}$ and usually stops. A possibility of a PT should be considered in a fibroadenoma that is bigger than this or continues to grow. The upper limit of FA size recommended for excision is between $2-3 \mathrm{~cm}$ in US (9), and $3 \mathrm{~cm}$ in the UK (5). The general recommendation is that FA be followed with ultrasound every 6 months for 2 years if managed conservatively and patient discharged at the end of this period if there no increase in size. In the UK 'One Stop Breast Clinics' follow up imaging is not routinely provided except in special circumstance. It is important that patients that are managed conservatively and discharged from follow up are aware of the small possibility of other pathologies that may mimic FA even after triple assessment and the need for continuous breast awareness.

\section{Conclusion}

4.3\% of FA excised in our specialist unit after Triple Assessment returns pathology other than FA post-excision, mostly benign phyllodes. FAs should be managed conservatively after triple assessment but surgery should be offered if there is still any doubt as there is no absolute guarantee that the presumed FA on core biopsy will remain a FA if excised. Also for medicolegal reasons and culture of openness, patients should be aware of this possibility especially if FA is to be managed conservatively.

\section{References}

[1] Kuijper A, Mommers EC, van der Wall E, van Diest PJ. Histopathology of fibroadenoma of the breast. Am J Clin Pathol. 2001; $115(5) \cdot 736-742$ https://doi.org/10.1309/F523-FMJV-W886-3J38.

[2] Dixon JM, Dobie V, Lamb J, Walsh JS, Chetty U. Assessment of the acceptability of conservative management of fibroadenoma of the breast. $\mathrm{Br} \quad \mathrm{J}$ Surg. 1996; 83:264-65. https://doi.org/10.1002/bjs.1800830238.

[3] Willett, A.M., Michell, M.J. and Lee, M.J.R. (2010) Best Practice Diagnostic Guidelines for Patients Presenting with Breast Symptoms. (Department of Health).

[4] Stavros AT, Thickman D, Rapp CL, Dennis MA, Parker SH, Sisney GA. Solid breast nodules: use of sonography to distinguish between benign and malignant lesions. Radiology 1995; 196(1):123-134). https://doi.org/10.1148/radiology.196.1.7784555. 
[5] London Cancer Surgical Guidelines for Breast Cancer http://www.londoncancer.org/media/134465/Surgical-Guidelinesfor-Breast-Cancer-November-2015.pdf.

[6] Dupont WD, Page DL, Parl FF, et al. Long-term risk of breast cancer in women with fibroadenoma. N Engl J Med. 1994; 331(1):10 15. [PubMed: 8202095] https://doi.org/10.1056/NEJM199407073310103.

[7] Nassar, A, Visscher D.W., MD, Degnim A.C. Complex Fibroadenoma and Breast Cancer Risk: A Mayo Clinic Benign Breast Disease Cohort Study. Breast Cancer Res Treat. 2015 September; 153(2): 397-405. https://doi.org/10.1007/s10549-015-3535-8.

[8] Lakhani SR, Ellis IO, Schnitt SJ, Tan, PH, van de Vijver MJ. World Health Organization Classification of Tumours of the Breast. Lyon: IARC Press; 2012. p. 143.

[9] Lee, S., Mercado, C.L., Cangiarella, J.F., and Chhor, C.M (2017). Frequency and oucomes of biopsy-proven fibroadenomas recommended for surgical excision. Clin. Imaging. 50:31-36 https://doi.org/10.1016/j.clinimag.2017.12.008.

[10] Yu-Ting Wu†, Shou-Tung Chen†, Chih-Jung Chen, Yao-Lung Kuo, Ling-Ming Tseng, Dar-Ren Chen, Shou-Jen Kuo and HungWen Lai. Breast cancer arising within fibroadenoma: collective analysis of case reports in the literature and hints on treatment policy. World Journal of Surgical Oncology 2014 12:335. https://doi.org/10.1186/1477-7819-12-335.

[11] Sanders, L.M. and Rana, S. (2015) The Growing Fibroadenoma.
Acta
Radiologica
Open,
4 ,
$1-5$. 